\title{
White Matter Alterations in Deficit Schizophrenia
}

\author{
Laura M Rowland*,', Elena A Spieker', Alan Francis', Peter B Barker ${ }^{2,3}$, William T Carpenter' and \\ Robert W Buchanan'
}

'Department of Psychiatry, Maryland Psychiatric Research Center, University of Maryland School of Medicine, Baltimore, MD, USA; ${ }^{2}$ Russell H Morgan Department of Radiology and Radiological Science, Johns Hopkins University School of Medicine, Baltimore, MD, USA; ${ }^{3}$ F.M. Kirby

Research Center for Functional Brain Imaging, Kennedy Krieger Institute, Baltimore, MD, USA

\begin{abstract}
Schizophrenia can be classified into two separate syndromes: deficit and nondeficit. Primary, enduring negative symptoms are used to define the deficit form of the illness, which is believed to have a unique neurobiological substrate. Previous research suggests that an aberrant prefrontal-thalamic-parietal network underlies deficit schizophrenia. In this study we conducted diffusion tensor imaging (DTI) fiber tracking to assess the integrity of the superior longitudinal fasciculus (SLF), the major white matter tract that connects prefrontal and parietal cortical regions, in deficit and nondeficit people with schizophrenia. We also used proton magnetic resonance spectroscopy ( $\left.{ }^{\prime} \mathrm{H}-\mathrm{MRS}\right)$ to assess neurochemistry in the left middle prefrontal and left inferior parietal cortical regions. A total of 20 subjects with schizophrenia (I0 deficit and 10 nondeficit) and II healthy subjects participated in this study. Results revealed reduced fractional anisotropy (FA), an index of white matter integrity, in the right hemisphere SLF and frontal white matter in the deficit subjects. There were no differences in MRS metabolite concentrations among groups. To our knowledge, this is the first DTI study to show compromised integrity of the major white matter tract that connects frontal and parietal regions in deficit schizophrenia. These findings provide further support for altered frontal-parietal network in deficit schizophrenia.

Neuropsychopharmacology (2009) 34, I5 I4- 1522; doi:I 0.1038/npp.2008.207; published online 3 December 2008
\end{abstract}

Keywords: deficit schizophrenia; DTI; SLF; fiber tracking; MRS

\section{INTRODUCTION}

The deficit form of schizophrenia is characterized by primary, enduring negative symptoms (Carpenter et al, 1985). Deficit schizophrenia may have a unique etiology, biological substrate, disease course, and possible treatment profile distinguishable from the nondeficit form (Carpenter et al, 1988; Kirkpatrick et al, 2001). The majority of neurobiological evidence, mostly from neuroimaging studies, suggests that an aberrant prefrontal-thalamic-parietal network underlies deficit schizophrenia. Blood flow studies using positron emission tomography (PET) and single photon emission computed tomography (SPECT) showed decreased prefrontal (Gonul et al, 2003; Heckers et al, 1999; Lahti et al, 2001 and parietal (Lahti et al, 2001) cortical blood flow in deficit compared to nondeficit subjects. A preliminary PET glucose metabolism study revealed lower frontal, thalamic, and parietal glucose metabolism in deficit compared to nondeficit subjects (Tamminga et al, 1992).

*Correspondence: Dr LM Rowland, Department of Psychiatry, Maryland Psychiatric Research Center, University of Maryland School of Medicine, P.O. Box 21247, Baltimore, MD 21228, USA, Tel: + | 410402 6803, Fax: + | 4104026077 ,

E-mail: Irowland@mprc.umaryland.edu

Received 8 May 2008; revised 20 October 2008; accepted 20 October 2008
Structural MRI studies findings are mixed. Compared to nondeficit subjects, deficit subjects showed right temporal lobe volume reductions (Galderisi et al, 2008) and bilateral frontal lobe increases (Buchanan et al, 1993). Compared to healthy control subjects, deficit subjects exhibited volume reductions in left superior temporal, left medial temporal, and medial frontal regions (Sigmundsson et al, 2001), or no brain volumetric differences (Buchanan et al, 1993; Gur et al, 1994). One study that investigated white matter volumes showed white matter reductions in the left temporal and frontal regions in deficit compared to healthy control subjects (Sigmundsson et al, 2001). In contrast, another study reported deficit subjects to have larger frontal white matter volumes than nondeficit subjects, but no differences existed when compared to healthy control subjects (Buchanan et al, 1993).

There are only two previous in vivo neurochemical investigations of deficit schizophrenia. One proton magnetic resonance $\left({ }^{1} \mathrm{H}-\mathrm{MRS}\right)$ study reported decreased left and right medial prefrontal cortical $\mathrm{N}$-acetylaspartate (NAA), a marker of neuronal integrity, in a small number of deficit $(n=5) v s$ nondeficit subjects $(n=17)$ (Delamillieure et al, 2000). In a second study, Sigmundsson et al (2003) reported no differences in dorsolateral cortical NAA in deficit schizophrenia $(n=25)$ compared to healthy control subjects $(n=26)$.

Only two post-mortem studies have investigated deficit schizophrenia. These studies showed increased interstitial 
white matter cell number in parietal (Kirkpatrick et al, 1999) and middle frontal (Kirkpatrick et al, 2003) regions of deficit schizophrenia compared to nondeficit and healthy control subjects. In combination with the functional imaging studies, these results support the involvement of the prefrontal and parietal heteromodal cortical regions in the neuroanatomy of the deficit form of schizophrenia, though structural MRI studies raise the question of potential temporal lobe involvement.

Diffusion tensor imaging (DTI) is a noninvasive neuroimaging technique that yields information about brain white matter integrity. It provides information about fiber tract organization and structure through quantification of the magnitude and direction of water diffusion in the brain. A common DTI measurement is fractional anisotropy (FA), an index of water diffusion ranging from 0 (isotropic) to 1 . Several DTI studies in schizophrenia revealed lower anisotropy in frontal white matter (Buchsbaum et al, 1998), splenium of the corpus callosum (Foong et al, 2000), and whole brain white matter (Lim et al, 1999). However, no studies of deficit $v s$ nondeficit schizophrenia have been published to date. DTI is an ideal technique to investigate the integrity of fiber tracts that connect the frontal and parietal regions, areas purported to be involved in deficit schizophrenia.

In this study, DTI fiber tracking was conducted to assess the integrity of the superior longitudinal fasciculus (SLF), the major white matter tract connecting frontal and parietal regions, in deficit and nondeficit schizophrenia. FA was also investigated within the middle frontal and parietal white matter regions. In the same subjects, proton magnetic resonance spectroscopy was also used ( ${ }^{1} \mathrm{H}$-MRS) to assess neurochemistry in the left middle prefrontal and left inferior parietal cortical regions.

We hypothesized that subjects with the deficit form of the illness would have compromised integrity of the SLF. This alteration would be reflected in reduced FA in the SLF. We predicted that FA in the white matter of the middle frontal, and parietal regions would also be reduced in the deficit group. Last, as deficit schizophrenia has been suggested to involve middle frontal and parietal cortices, we predicted that deficit patients would have (1) compromised neuronal integrity, as reflected by reduced NAA, and (2) altered glutamatergic metabolism, as reflected by reduced glutamate and glutamine (GLE), in the left middle frontal and left inferior parietal cortices.

\section{METHODS}

\section{Course of Events}

This study was conducted over two sessions separated by one to two weeks. Psychiatric and neuropsychological assessments were conducted during the first session and MR imaging was conducted during the second session. Patients were evaluated for psychopathology with the Brief Psychiatric Rating Scale (BPRS; Overall and Gorham, 1962) and the Scale for the Assessment of Negative Symptoms (SANS; Andreasen, 1984). All subjects completed the Repeatable Battery for the Assessment of Neuropsychological Status (RBANS; Gold et al, 1999; Randolph, 1988) as a measure of general cognitive function. Session 1 took about
1-2 $\mathrm{h}$ and was completed within approximately 1 week of MRS/DTI scanning. Subjects were paid $\$ 10.00$ per hour for their time. Session 2 took approximately $1 \mathrm{~h}$ and subjects were paid $\$ 100$ for their time.

\section{Subjects}

A total of 20 outpatients with chronic schizophrenia treated with antipsychotic medication (10 deficit and 10 nondeficit) and 11 healthy volunteers participated in this study. The inclusion/exclusion criteria for volunteers with schizophrenia were: (1) diagnosis of schizophrenia as determined with the Structured Clinical Interview for DSM-IV, Patient Version (SCID-P; First et al, 1995), (2) diagnosis of deficit or nondeficit schizophrenia as determined by the Schedule for Deficit Syndrome (Kirkpatrick, 1989); (3) no current or past neurological condition, (4) no DSM-IV substance abuse or dependence (other than nicotine) in the past 6 months, (5) clinically stable as determined by their treatment psychiatrist, and (6) right-handed. The inclusion/exclusion criteria for healthy volunteers were: (1) no past or present psychiatric disorder as determined with the Structured Clinical Interview for DSM-IV, Non-Patient Version (SCIDNP; First et al, 1995), (2) no first-degree relatives with a diagnosis of a psychotic disorder, (3) no current or past neurological condition, (4) no DSM-IV substance abuse or dependence (other than nicotine) in the past 6 months, and (5) right-handed. Volunteers with schizophrenia were evaluated for their ability to provide informed consent before signing consent documents. All subjects gave written informed consent before participation in the study. This study was approved by the University of Maryland and Johns Hopkins University Internal Review Committees.

\section{MR Imaging}

MR scanning was conducted on a Philips Intera 3T MR scanner using a six-channel head coil (Philips Medical Systems, Best, The Netherlands). DTI data were acquired with a spin echo single shot, echo-planar imaging sequence with sensitivity encoding (SENSE $=2.5)$ encoding $(2.2 \mathrm{~mm}$ isotropic voxels, $212 \times 212 \mathrm{~mm}$ FOV, $96 \times 96$ acquired matrix), TR/TE $=6338 / 69 \mathrm{~ms}, 60$ slices for whole brain coverage, with diffusion gradients applied along 32 noncollinear directions at a $b$ factor of $700 \mathrm{~s} / \mathrm{mm}^{2}$. A minimally weighted image with $b=0 \mathrm{~s} / \mathrm{mm}^{2}$ was also acquired. Anatomical $\mathrm{T}_{1}$-weighted images were collected with a 'MPRAGE' sequence (SENSE factor 2, $1 \mathrm{~mm}$ isotropic voxels, $256 \times 256 \mathrm{~mm}$ FOV, TR/TE/TI $=8 / 3.8 / 842.5 \mathrm{~ms}$, flip angle $=8^{\circ}$ ). Diffusion weighted and MPRAGE images were co-registered to the minimally diffusion weighted image $\left(b=0 \mathrm{~s} / \mathrm{mm}^{2}\right.$ ) with 'FLIRT' (www.fmrib.ox.ac.uk/fsl/flirt; Jenkinson and Smith, 2001) using a 6 degrees of freedom (rigid body) model and correlation ratio cost function. The DTI gradient table, which specifies the orientation of the diffusion weighting vector for each diffusion weighted image, was corrected for slice angulation and the rotational component of image co-registration (Farrell et al, 2007). The co-registered diffusion weighted and MPRAGE anatomical images were used for all analyses.

Spectra were acquired with a point resolved pulse sequence ('PRESS', TR $=2000 \mathrm{~ms}, \mathrm{TE}=35 \mathrm{~ms}, 1024$ points, 
$2000 \mathrm{~Hz}$ spectral width, 128 averages water suppressed and 16 averages without water suppression) from $1.5 \times 1.5 \times 1.5 \mathrm{~cm}$ voxels placed in the left middle frontal and left inferior parietal regions. See Figure 1 for illustration of voxel placement and representative spectra. Voxels were placed in a manner to maximize the gray matter volume and minimize white matter and CSF. Water suppression was achieved using three chemical-shift selected (CHESS)

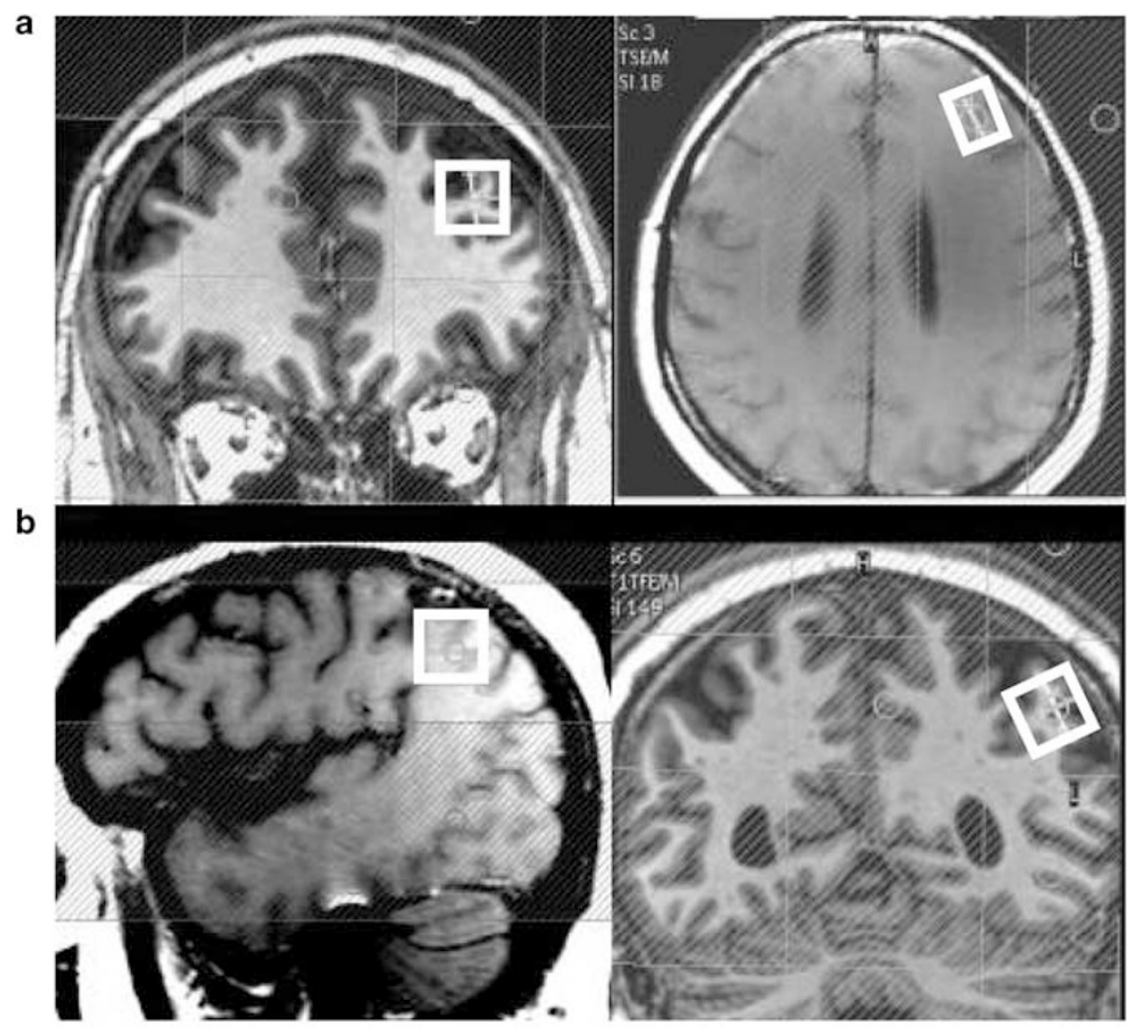

C

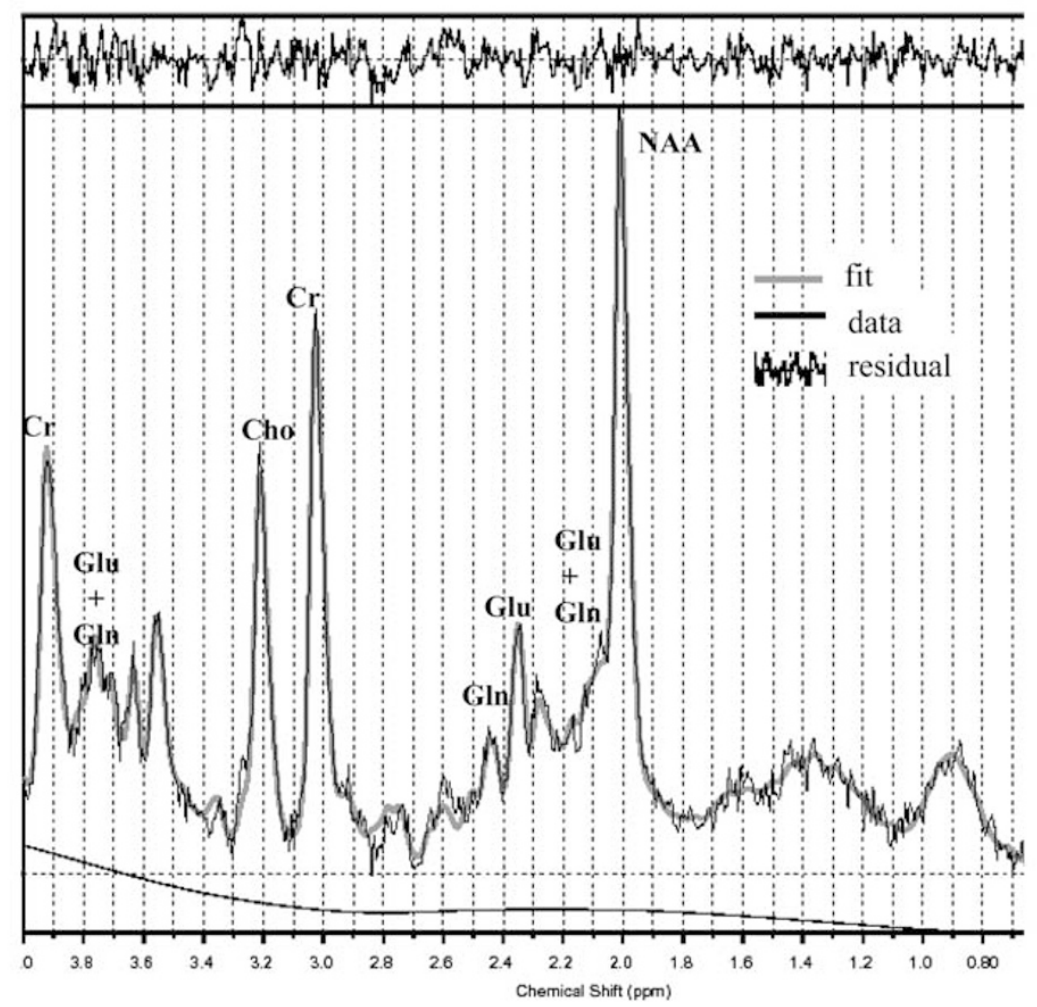

Figure I Illustration of spectroscopic voxel $\left(1.5 \mathrm{cc}^{3}\right)$ placement for left middle frontal region (a) and left inferior parietal region (b) and representative spectra (c). 
presaturation pulses. All sequences fell within FDA guideline SAR limits of $3.0 \mathrm{~W} / \mathrm{kg}$ average power deposition for head imaging.

Spectra were analyzed using fully automated curve fitting software, 'LCModel' (Provencher, 2001). Spectra were normalized to the unsuppressed water signal, yielding quantification of NAA, glutamate + glutamine (GLE), choline-containing compounds (Cho), and creatine $(\mathrm{Cr})$ in $\mathrm{mM}$ quantities. Metabolite concentration uncertainties that exceeded a Cramer-Rao bounds of $20 \%$ were considered poor quality and were excluded from further analyses. Spectra were also considered poor quality and rejected from further analyses if the metabolite linewidth (full width half maximum) exceeded 0.1 p.p.m., or if obvious movement artifact was observed by visual inspection.

\section{Fiber Tractography}

Analysis of the DTI data (ie computation of FA maps, color maps, and fiber tracking) was performed using DTIStudio (Jiang et al, 2006). To segment SLF fibers, one seed region of interest (ROI) was drawn around the SLF on the coronal color map image at the level of the posterior portion of the putamen (Wakana et al, 2004). The SLF fibers in the left and right hemispheres were delineated separately. 3D tract reconstruction was automatically computed in DTIStudio using the Fiber Assignment by Continuous Tracking algorithm (FACT), with stopping criteria being a FA threshold of less than 0.25 , or a change in fiber angle from one pixel to the next of $70^{\circ}$ or greater. The raters then eliminated those fibers that did not anatomically fall within the SLF by overlaying each individual's SLF fiber tract onto the corresponding co-registered MPRAGE anatomical image. The elimination of fibers falling outside the SLF was achieved through visual inspection, and using a white matter atlas as a guide (Mori et al, 2005). See Figure 2 for illustration of SLF fiber tracking.

To determine the reliability of SLF fiber tracking, two raters completed measurements on five brains. The interrater reliability was good with the intraclass correlations (ICCs) greater than 0.90 for the SLF fiber tract characteristics (ie mean fiber length, volume, FA). After good interrater reliability was established, one rater (RWB) completed all measurements.

\section{Frontal and Parietal FA}

Volumetric white matter values were obtained through manual ROI measurements drawn in the middle frontal (MF) by EAS and inferior parietal (IP) by AF on MPRAGE anatomical images that were co-registered to the DTI $b=0 \mathrm{~s} / \mathrm{mm}$ image. White matter was segmented through manual ROI tracings within DTIstudio for each individual subject. These ROIs were then overlaid on the FA images to obtain FA only within these white matter ROIs. The method for middle frontal and inferior parietal volumetric measurements were similar to Buchanan et al $(1998,2004)$. Interrater reliability was good (ICCs $>0.9$ ). To obtain FA values, the white matter ROIs were overlaid on FA images created from the co-registered DTI images in DTIStudio. Both hemispheres were examined.
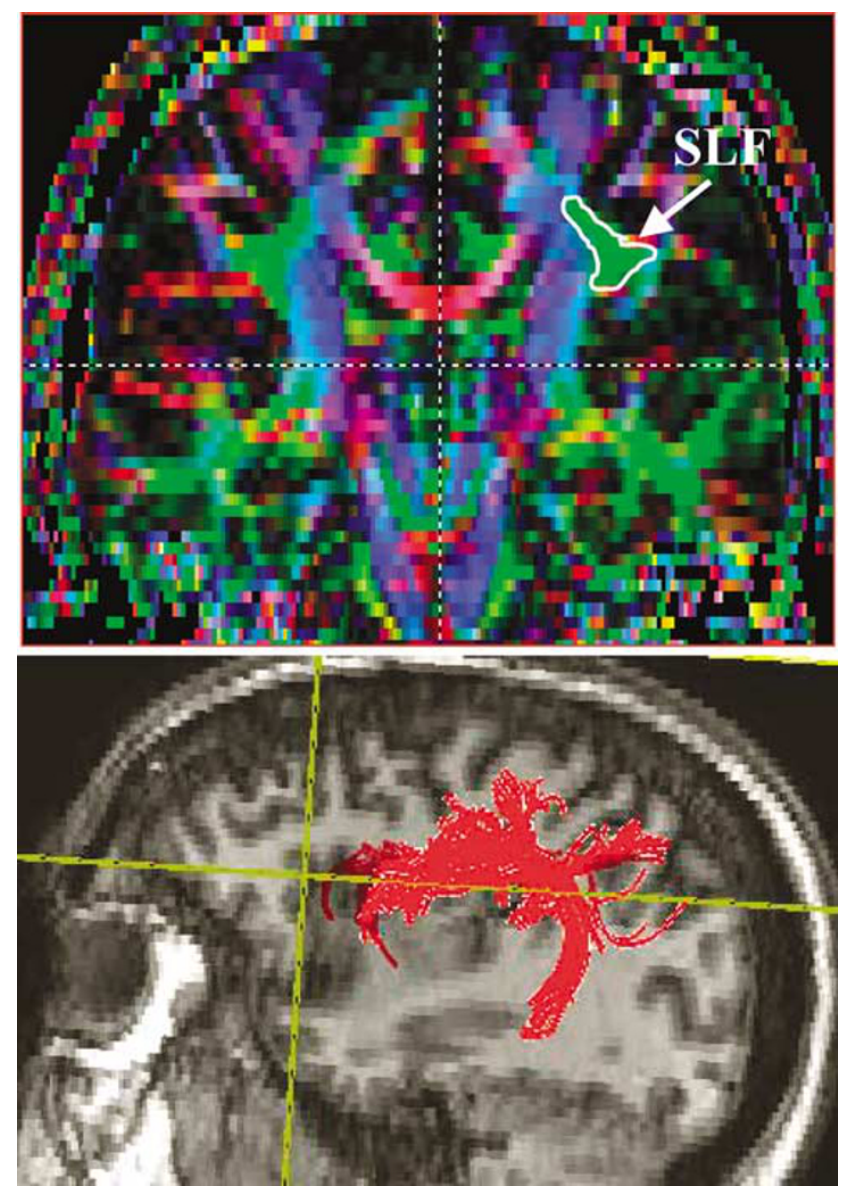

Figure 2 Illustration of superior longitudinal fasiculus (SLF) fiber tracking method. Top panel shows the region used for seeding the fiber tracking algorithm, whereas the bottom panel shows a $3 D$ representation of the fiber tracking results.

\section{Data Analyses}

DTI and ${ }^{1} \mathrm{H}$-MRS measures were analyzed with one-way ANOVA with $\alpha=0.05$. Owing to the preliminary nature of this study, DTI measures were analyzed separately per hemisphere and statistically significant effects were followed up with post hoc tests, not corrected for multiple comparisons. The relationships between DTI and ${ }^{1} \mathrm{H}-\mathrm{MRS}$ measures and psychiatric ratings were computed with Pearson's product moment correlations. Analyses were conducted with the Statistical Package for Social Sciences (SPSS) version 12.0 software package.

\section{RESULTS}

Subject demographic and clinical characteristics are given in Table 1. As expected, there was a significant difference in years of education amongst groups $(\mathrm{F}(2,28)=7.0, p<0.01)$; post hoc tests revealed less years of education in deficit subjects compared to healthy controls $(p<0.05)$. Also as expected, there was a significant difference in RBANS scores amongst groups $(\mathrm{F}(2,28)=6.2, p<0.01)$; post hoc tests revealed lower RBANS scores in both deficit and nondeficit subject groups compared to healthy controls $(p<0.01)$. 
There were no significant differences in age, duration of illness, gender, or ethnicity between groups (all $p$ 's $>0.05$ ). The deficit group had significantly higher SANS scores compared to the nondeficit group $(\mathrm{t}(18)=2.9, p<0.05)$. In addition to not meeting DSM-IV criteria for substance abuse or dependence (other than nicotine) in the past 6 months, no deficit subjects met lifetime criteria for alcohol/ substance dependence and only 1 nondeficit subject met criteria for lifetime substance dependence (marijuana), which resolved over 4 years before the study scans. In terms of alcohol/substance abuse, two deficit and three nondeficit subjects met criteria for lifetime alcohol abuse, and one deficit and three nondeficit subjects met criteria for lifetime substance abuse. All of these subjects, except for one nondeficit subject, had not abused alcohol or substances for at least 5 years before their scan. The one nondeficit subject had their alcohol abuse resolve 2.5 years before the study scans. The numerically greater lifetime

Table I Subject Characteristics

\begin{tabular}{|c|c|c|c|}
\hline & $\begin{array}{c}\text { Deficit } \\
(n=10)\end{array}$ & $\begin{array}{l}\text { Nondeficit } \\
(n=10)\end{array}$ & $\begin{array}{l}\text { Control } \\
(n=I I)\end{array}$ \\
\hline \multicolumn{4}{|l|}{ Gender } \\
\hline Male & 8 & 8 & 8 \\
\hline Female & 2 & 2 & 3 \\
\hline Age (years) & $43(6)$ & $40(6)$ & $37(10)$ \\
\hline \multicolumn{4}{|l|}{ Ethnicity } \\
\hline Caucasian & 7 & 4 & 6 \\
\hline African American & 3 & 6 & 4 \\
\hline Hispanic & 0 & 0 & I \\
\hline Education (years) & $12(2.3)^{*}$ & $13(1.6)$ & I5 (1.7) \\
\hline RBANS & $74.8(14)^{*}$ & $77(16)^{*}$ & $95.5(14)$ \\
\hline $\begin{array}{l}\text { Duration of illness } \\
\text { (years) }\end{array}$ & $24(8)$ & $19(7)$ & \\
\hline \multicolumn{4}{|l|}{ Psychiatric ratings } \\
\hline SANS & $36.4(13.6)^{+}$ & $21.3(9.7)$ & \\
\hline BPRS (total) & $37.1(7.6)$ & $34.2(9.4)$ & \\
\hline BPRS (+ subscale) & $10.6(4.9)$ & $10.4(4.6)$ & \\
\hline BPRS (- subscale) & $10.4(4.4)$ & $7.5(3.7)$ & \\
\hline \multicolumn{4}{|l|}{ Antipsychotic medications } \\
\hline Olanzapine & 4 & 3 & \\
\hline Risperidone & 2 & I & \\
\hline Fluphenazine & 3 & 0 & \\
\hline Clozapine & 0 & 2 & \\
\hline Clozapine+Risperdal & । & 2 & \\
\hline Clozapine+Seroquel & 0 & I & \\
\hline Haloperidol & 0 & । & \\
\hline $\begin{array}{l}\text { Mean (SD). } \\
*^{*} p<0.05 \text {, vs control. } \\
{ }^{+} p<0.05 \text {, vs nondeficit. }\end{array}$ & & & \\
\hline
\end{tabular}

Neuropsychopharmacology history of substance/alcohol abuse/dependence disorders in the nondeficit subjects is consistent with previous studies of substance/alcohol abuse and dependence in deficit and nondeficit people with schizophrenia (Kirkpatrick et al, 1996). No additional differences in psychiatric symptoms between the deficit and nondeficit groups were observed.

\section{SLF Fiber Tracking}

Fiber tracking and FA measures were not completed for one subject with deficit schizophrenia due to motion artifact. There was a significant group difference in FA for the right hemisphere SLF $(\mathrm{F}(2,27)=3.6, p=0.04$; Cohen's $d$ effect size $=1.1)$. Post hoc analyses revealed lower SLF FA in the deficit group compared to the healthy control group $(p<0.05)$. The deficit group had lower SLF FA compared to the nondeficit group with the difference approaching statistical significance $(p=0.07 ;$ Cohen's $d$ effect size $=0.86$ ). The nondeficit group did not significantly differ from the healthy control group. Left hemisphere SLF FA values were not significantly different among the groups. SLF measures were not related to age, psychiatric symptoms or RBANS scores (all $p$ 's $>0.05$ ). Descriptive statistics for the SLF FA values are presented in graphical form in Figure 3.

\section{Middle Frontal and Inferior Parietal Region Measures}

Descriptive statistics and $p$-values for both middle frontal and inferior parietal measures are presented in Table 2. White matter volume for middle frontal and inferior parietal regions did not significantly differ among groups (all $p$ 's $>0.05$ ). With respect to FA values, only the FA in the right hemisphere middle frontal region significantly differed among groups $(\mathrm{F}(2,27)=7.4, p<0.01)$. Post hoc analyses revealed higher FA in the healthy control group compared to the deficit $(p=0.00$; Cohen's $d$ effect size $=1.9)$ and nondeficit $(p=0.037$, Cohen's $d$ effect size $=1.0)$ groups. The deficit group had a trend $(p=0.1$; Cohen's $d$ effect size $=0.75$ ) towards lower FA compared to

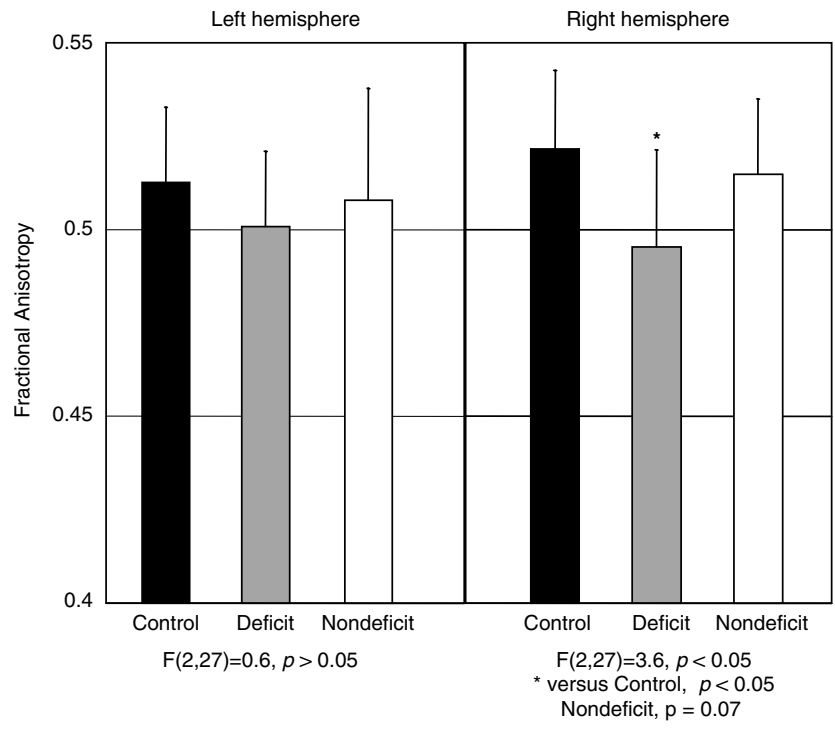

Figure 3 Superior longitudinal fasciculus fractional anisotropy values. 
the nondeficit group. FA in the right inferior parietal region approached a significant difference among groups $(\mathrm{F}(2,27)=3.0, p=0.07)$, with the deficit group having the lowest FA values. Middle frontal and inferior parietal volume and FA measures were not related to positive or negative symptoms or RBANS scores (all p's $>0.05$ ).

Middle frontal volume and FA were significantly related to age (right volume: $r=-0.4$; left volume: $r=-0.38$, right FA: $r=-0.42$, left FA: $r=-0.47$ ( $p$ 's $<0.05)$. However, when age was included as a covariate, the outcome of the statistical analyses did not change. Inferior parietal volume and FA measures were not related to age.

\section{${ }^{1}$ H-MRS}

Spectra were not available from three subjects due to either poor quality (one healthy control, one nondeficit subject) or to severe movement halting spectra data acquisition (one deficit subject). Contrary to expectations, there were no statistically significant differences between groups for any spectroscopic measures for the left middle frontal and left inferior parietal regions (all $p$ 's $>0.05$ ). Spectroscopic measures were not related to age, positive or negative symptoms, RBANS scores, or FA measures (all p's >0.05). Descriptive statistics and $p$-values are presented in Table 3.

\section{DISCUSSION}

To our knowledge, this is the first study to use DTI to investigate the white matter integrity of the SLF, middle frontal, and inferior parietal regions in deficit and nondeficit schizophrenia. We demonstrate a reduction in FA of the right hemispheric SLF and middle frontal region in deficit schizophrenia compared to healthy control subjects. The differences between deficit and nondeficit groups on these measures approached statistical significance. There was no significant difference between nondeficit and healthy control groups in SLF FA, but middle frontal FA was lower in nondeficit compared to healthy controls. The finding of reduced middle frontal FA in schizophrenia is a consistent finding (for review see Kubicki et al, 2007).

To our knowledge, only one other DTI tractography study on the SLF has been conducted in schizophrenia (Jones et al, 2006). Our finding of reduced FA in the right SLF in deficit subjects is partially consistent with this study, in which they showed reduced FA in the SLF in schizophrenia (Jones et al, 2006). Owing to the small sample size, the study by Jones et al (2006) was not able to investigate the differences between subjects based on clinical characteristics. Therefore, it is possible that the FA reduction in the SLF is more prominent in those people with primary enduring negative symptoms as we have observed.

Reduced FA could indicate impaired myelination, altered fiber organization, or aberrant axon morphology. It is unlikely that reduced FA reflects demyelination because there does not appear to be an active deteriorating white matter process in schizophrenia similar to that observed in other diseases such as multiple sclerosis. In addition, we did not observe any white matter volume alterations across the three groups. Rather, reduced FA may reflect dysmyelination, or problems with the production or structure of myelin. Post-mortem evidence supports this notion. Ultrastructural abnormalities in myelin (Uranova et al, 1996, 2001), and alterations in oligodendrocyte density or number (Hof et al, 2003; Uranova et al, 2004; Vostrikov et al, 2007) have been reported in schizophrenia. It is also plausible that reduced FA reflects aberrant fiber orientation or axon morphology. Post-mortem research has revealed atrophy of axons in schizophrenia (Uranova et al, 2007). Reduced FA in the middle frontal region and SLF could also reflect the post-mortem findings of increased interstitial cells of the white matter in deficit compared to nondeficit schizophrenia (Kirkpatrick et al, 1999, 2003). Although we are unable to pinpoint the exact pathological process occurring

Table 3 Left Middle Frontal and Left Inferior Parietal 'H-MRS Measures

\begin{tabular}{lrrrr}
\hline & Control & Deficit & Nondeficit & $\boldsymbol{p}$ \\
\hline Left middle frontal (mean (SD))* & & & \\
NAA & $8.7(0.6)$ & $8.7(1.7)$ & $8.2(1.0)$ & 0.5 \\
GLE (glu+gln) & $10.7(2.5)$ & $11.1(2.4)$ & $9.6(2.0)$ & 0.4 \\
Cho & $1.8(0.2)$ & $1.7(0.4)$ & $1.9(0.2)$ & 0.5 \\
Cr & $7.7(0.7)$ & $7.4(1.4)$ & $7.3(0.4)$ & 0.5 \\
& & & & \\
Left inferior parietal (mean (SD))* & & & \\
NAA & $8.9(1.5)$ & $8.2(0.7)$ & $8.5(1.3)$ & 0.5 \\
GLE (glu+gln) & II.0(I.9) & $11.6(1.9)$ & $11.4(1.6)$ & 0.6 \\
Cho & $1.6(0.3)$ & $1.7(0.3)$ & $1.9(0.2)$ & 0.1 \\
Cr & $7.5(0.8)$ & $7.0(1.0)$ & $7.8(1.3)$ & 0.3 \\
\hline
\end{tabular}

*Metabolites are presented in institutional values.

Table 2 Middle Frontal (MF) and Inferior Parietal (IP) White Matter Volume and Fractional Anisotropy (FA)

\begin{tabular}{|c|c|c|c|c|c|c|c|c|}
\hline & \multicolumn{4}{|c|}{ Left hemisphere, mean (SD) } & \multicolumn{4}{|c|}{ Right hemisphere, mean (SD) } \\
\hline & Control & Deficit & Nondeficit & $p$ & Control & Deficit & Nondeficit & $p$ \\
\hline MF volume $\left(\mathrm{cm}^{3}\right)$ & $10.7(1.7)$ & $9.2(3.0)$ & $11.0(3.1)$ & 0.31 & | $1.8(2.2)$ & |0.1 (3.5) & | $1.3(3.0)$ & 0.43 \\
\hline IP FA & $0.38(0.09)$ & $0.39(0.03)$ & $0.39(0.02)$ & 0.76 & $0.40(0.03)$ & $0.37(0.04)$ & $0.39(0.04)$ & 0.07 \\
\hline
\end{tabular}

*vs control, $p<0.05$ 
in white matter in schizophrenia, it is likely that reduced FA in the right middle frontal region and SLF reflects compromised white matter connectivity in deficit schizophrenia.

Reduced FA in deficit subjects was confined to the right hemisphere. There are several studies that complement this lateralized finding. Some MEG and EEG studies revealed a relationship between negative symptoms and reduced right hemisphere M50 sensory gating (Thoma et al, 2005), and reduced EEG- $\alpha$ coherence between right hemisphere frontal and parietal regions (Merrin and Floyd, 1996). Consistently, subjects with predominantly negative symptoms have lower right hemisphere glucose metabolism during a continuous performance task (Potkin et al, 2002), and abnormal right hemisphere frontal and parietal regional cerebral blood flow (rCBF) during word production tasks (Artiges et al, 2000). Lahti et al (2001) observed reduced rCBF specifically in right hemisphere middle frontal and inferior parietal regions during an effortful auditory discrimination task in deficit $v s$ nondeficit subjects. A recent structural MRI study found less right temporal lobe volume in deficit subjects compared to nondeficit subjects (Galderisi et al, 2008). The right hemisphere is more specialized than the left hemisphere in emotion perception and interpretation (for review see Kucharska-Pietura, 2006; Pell, 2006). People with schizophrenia show emotional processing and interpretation impairments more comparable to people with rightsided hemisphere damage than those with left-sided hemisphere damage (for review see Kucharska-Pietura, 2006; Blake, 2003; Mitchell and Crow, 2005). People with the deficit form of schizophrenia are more severely impaired in both emotional expression and perception than people with the nondeficit form of the illness. Therefore, this may help to explain our and other researchers observations of right hemisphere brain alterations in people with greater negative symptoms.

White matter alterations observed in the deficit group were not related to positive or negative symptoms. This may indicate that the relationship between white matter alterations and negative symptoms is more complicated than a linear pattern, or due to the preliminary nature of this study (ie underpowered). There have been very few studies that revealed a relationship between DTI indices and psychiatric symptoms in schizophrenia. There are reports of correlations between negative symptoms and DTI indices within white matter regions near the insula (Skelly et al, 2008; Shin et al, 2006) and inferior frontal regions (Wolkin et al, 2003). However, the majority of DTI studies have not found a relationship between psychiatric symptoms and DTI indices in schizophrenia (for review see Kubicki et al, 2007).

We did not find any significant differences in spectroscopic measures between groups. There are several plausible reasons for the negative findings. First, there may be no spectroscopic differences between deficit and nondeficit schizophrenia in the middle frontal and inferior parietal cortical regions. Our middle frontal region negative findings are consistent with a larger study demonstrating no NAA, Cho, or $\mathrm{Cr}$ differences in the middle frontal cortex in deficit and nondeficit schizophrenia (Sigmundsson et al, 2003). Second, it is possible that differences may lie in different cortical regions, as Delamillieure et al (2000) found NAA differences in a medial frontal region in a small number of deficit subjects compared to nondeficit subjects. Third, due to time constraints we were not able to assess the right hemisphere. It is possible that MRS differences lie within the right hemisphere in deficit schizophrenia, similar to the DTI differences observed in this study. Last, MRS differences may be much more pronounced in white matter in deficit schizophrenia. This would be consistent with our DTI findings.

There are several limitations of this study. First, the results are preliminary. Data must be acquired on more subjects to substantiate these findings. Second, spectra should be acquired from frontal and parietal regions in both hemispheres. It is possible that middle frontal and inferior parietal cortical NAA and GLE are reduced in the right hemisphere in deficit subjects. Proton spectra should also be acquired from white matter regions to determine if neurochemical alterations coincide with SLF and middle frontal FA reductions. A control region that falls outside the SLF and frontal and parietal regions for white matter FA should be investigated to determine the regional specificity of these findings. Last, we cannot fully rule out the possibility of an antipsychotic effect on FA. However, both the deficit and nondeficit groups were comparable with respect to the type of medications used.

In summary, we present evidence for compromised integrity of right hemisphere SLF and middle frontal white matter in deficit schizophrenia. These findings indicate that the major white matter connection between frontal and parietal regions is likely disrupted and contributes to the deficit form of schizophrenia. Future studies should further consider the impact of primary, enduring negative symptoms in schizophrenia on DTI indices.

\section{ACKNOWLEDGEMENTS}

This study was supported in part by NIMH grants P30 068580 (Robert W Buchanan) and K01MH077230 (Laura M Rowland). We thank the volunteers, especially the patients, for participating in the study. We thank Terri Brawner, Kathleen Kahl, and Ivana Kusevic for conducting the MR scans. We thank Craig Jones and Jonathan Farrell for their valuable help and guidance with the DTI methods and analyses.

\section{DISCLOSURE/CONFLICT OF INTEREST}

Dr Barker, Dr Rowland, Dr Francis, and Ms Spieker declare that, except for income received from my primary employer, no financial support or compensation has been received from any individual or corporate entity over the past three years for research or professional service and there are no personal financial holdings that could be perceived as constituting a potential conflict of interest. Dr Carpenter has no conflicts of interest for the current project to report. He has no stock holdings or options in commercial entities, which market medical products; he does not participate in any speakers bureau, and receives no grant funding from industry. During 2006-2008 he has served as an advisor or prepared educational material for the following companies: Merck, McNeil, Janssen, Pfizer, Solvey/Wyeth, Lilly, Cephalon, and Tava. Income from these sources is less than $5 \%$ of professional income, which comes from the University of Maryland, Baltimore, supported in turn from NIMH and 
NARSAD. He holds an European Regional Patent Number 1487998 (6 June 2007) 'Methods for Diagnosing and Treating Schizophrenia' with no potential for personal financial gain (any income pledged to MPRC). Dr Buchanan has no conflicts of interest for the current project to report. He has no stock holdings or options in commercial entities, which market medical products, and he does not participate in any speakers bureau. He has received the following research support from industry: Ortho-McNeil supplied study medications for a foundation supported clinical trial of galantamine, an acetylcholinesterase inhibitor and Janssen supplied study medications for a NIMH-supported clinical trial of risperidone. He has served on advisory boards for Astra-Zeneca, Merck, Pfizer, Solvey/Wyeth, as a consultant for Natixis Bleichroeder, Organon, Glaxo-SmithKline, Sanofi-Aventis, and on data safety monitoring boards for Pfizer and Wyeth.

\section{REFERENCES}

Andreasen NC (1984). The Scale for the Assessment of Negative Symptoms. University of Iowa: Iowa City.

Artiges E, Martinot JL, Verdys M, Attar-Levy D, Mazoyer B, Tzourio $\mathrm{N}$ et al (2000). Altered hemispheric functional dominance during word generation in negative schizophrenia. Schizophr Bull 26: 709-721.

Blake ML (2003). Affective language and humor appreciation after right hemisphere brain damage. Semin Speech Lang 24: 107-119.

Buchanan RW, Breier A, Kirkpatrick B, Elkashef A, Munson RC, Gellad F et al (1993). Structural abnormalities in deficit and nondeficit schizophrenia. Am J Psychiatry 150: 59-65.

Buchanan RW, Francis A, Arango C, Miller K, Lefkowitz DM, McMahon RP et al (2004). Morphometric assessment of the heteromodal association cortex in schizophrenia. Am J Psychiatry 161: 322-331.

Buchanan RW, Vladar K, Barta RE, Pearlson GD (1998). Structural evaluation of the prefrontal cortex in schizophrenia. $\mathrm{Am} \mathrm{J}$ Psychiatry 155: 1049-1055.

Buchsbaum MS, Tang CY, Peled S, Gudbjartsson H, Lu D, Hazlett EA et al (1998). MRI white matter diffusion anisotropy and PET metabolic rate in schizophrenia. Neuroreport 9: 425-430.

Carpenter Jr WT, Heinrichs DW, Alphs LD (1985). Treatment of negative symptoms. Schizophr Bull 11: 440-452.

Carpenter Jr WT, Heinrichs DW, Wagman AM (1988). Deficit and nondeficit forms of schizophrenia: the concept. Am J Psychiatry 145: $578-583$.

Delamillieure P, Fernandez J, Constans JM, Brazo P, Benali K, Abadie $\mathrm{P}$ et al (2000). Proton magnetic resonance spectroscopy of the medial prefrontal cortex in patients with deficit schizophrenia: preliminary report. Am J Psychiatry 157: 641-643.

Farrell JA, Landman BA, Jones CK, Smith SA, Prince JL, van Zijl PC et al (2007). Effects of signal-to-noise ratio on the accuracy and reproducibility of diffusion tensor imaging-derived fractional anisotropy, mean diffusivity, and principal eigenvector measurements at 1.5 T. J Magn Reson Imaging 26: 756-767.

First MB, Spitzer RL, Gibbon M, Williams JBW (1995). Structured Clinical Interview for DSM-IV Axis I Disorders, Non-Patient Edition (SCID-NP), version 2. New York State Psychiatric Institute, Biometrics Research: New York.

Foong J, Maier M, Clark CA, Barker GJ, Miller DH, Ron MA (2000). Neuropathological abnormalities of the corpus callosum in schizophrenia: a diffusion tensor imaging study. J Neurol Neurosurg Psychiatry 68: 242-244.

Galderisi S, Quarantelli M, Volpe U, Mucci A, Cassano GB, Invernizzi $G$ et al (2008). Patterns of structural MRI abnorm- alities in deficit and nondeficit schizophrenia. Schizophr Bull 34: 393-401.

Gold JM, Queern C, Iannone VN, Buchanan RW (1999). Repeatable battery for the assessment of neuropsychological status as a screening test in schizophrenia I: sensitivity, reliability, and validity. Am J Psychiatry 156: 1944-1950.

Gonul AS, Kula M, eg;el E, u ${ }^{\circ}$ A, Sofuoglu S (2003). A Tc-99m HMPAO SPECT study of regional cerebral blood flow in drugfree schizophrenic patients with deficit and non-deficit syndrome. Psychiatry Res 123: 199-205.

Gur RE, Mozley PD, Shtasel DL, Cannon TD, Gallacher F, Turetsky B et al (1994). Clinical subtypes of schizophrenia: differences in brain and CSF volume. Am J Psychiatry 151: 343-350.

Heckers S, Goff D, Schacter DL, Savage CR, Fischman AJ, Alpert NM et al (1999). Functional imaging of memory retrieval in deficit vs nondeficit schizophrenia. Arch Gen Psychiatry 56: 1117-1123.

Hof PR, Haroutunian V, Friedrich Jr VL, Byne W, Buitron C, Perl DP et al (2003). Loss and altered spatial distribution of oligodendrocytes in the superior frontal gyrus in schizophrenia. Biol Psychiatry 53: 1075-1085.

Jenkinson M, Smith S (2001). A global optimisation method for robust affine registration of brain images. Med Image Anal 5: $143-156$.

Jiang H, van Zijl PC, Kim J, Pearlson GD, Mori S (2006). DtiStudio: resource program for diffusion tensor computation and fiber bundle tracking. Comput Methods Programs Biomed 81: 106-116.

Jones DK, Catani M, Pierpaoli C, Reeves SJ, Shergill SS, O'Sullivan $M$ et al (2006). Age effects on diffusion tensor magnetic resonance imaging tractography measures of frontal cortex connections in schizophrenia. Hum Brain Mapp 27: 230-238.

Kirkpatrick B, Amador XF, Flaum M, Yale SA, Gorman JM, Carpenter Jr WT et al (1996). The deficit syndrome in the DSMIV field trial: I. Alcohol and other drug abuse. Schizophr Res 20: 69-77.

Kirkpatrick B, Buchanan RW, McKenney PD, Alphs LD, Carpenter Jr WT (1989). The Schedule for the Deficit syndrome: an instrument for research in schizophrenia. Psychiatry Res 30: 119-123.

Kirkpatrick B, Buchanan RW, Ross DE, Carpenter Jr WT (2001). A separate disease within the syndrome of schizophrenia. Arch Gen Psychiatry 58: 165-171.

Kirkpatrick B, Conley RC, Kakoyannis A, Reep RL, Roberts RC (1999). Interstitial cells of the white matter in the inferior parietal cortex in schizophrenia: an unbiased cell-counting study. Synapse 34: 95-102.

Kirkpatrick B, Messias NC, Conley RR, Roberts RC (2003). Interstitial cells of the white matter in the dorsolateral prefrontal cortex in deficit and nondeficit schizophrenia. J Nerv Ment Dis 191: 563-567.

Kubicki M, McCarley R, Westin CF, Park HJ, Maier S, Kikinis R et al (2007). A review of diffusion tensor imaging studies in schizophrenia. J Psychiatr Res 41: 15-30.

Kucharska-Pietura K (2006). Disordered emotional processing in schizophrenia and one-sided brain damage. Prog Brain Res 156: 467-479.

Lahti AC, Holcomb HH, Medoff DR, Weiler MA, Tamminga CA, Carpenter Jr WT (2001). Abnormal patterns of regional cerebral blood flow in schizophrenia with primary negative symptoms during an effortful auditory recognition task. Am J Psychiatry 158: $1797-1808$.

Lim KO, Hedehus M, Moseley M, de Crespigny A, Sullivan EV, Pfefferbaum A (1999). Compromised white matter tract integrity in schizophrenia inferred from diffusion tensor imaging. Arch Gen Psychiatry 56: 367-374.

Merrin EL, Floyd TC (1996). Negative symptoms and EEG alpha in schizophrenia: a replication. Schizophr Res 19: 151-161. 
Mitchell RL, Crow TJ (2005). Right hemisphere language functions and schizophrenia: the forgotten hemisphere. Brain 128: 963-978.

Mori S, Wakana S, Nagae-Poetscher LM, van Zil PCM (2005). MRI Atlas of Human White Matter. Elsevier B.V.: Amsterdam, The Netherlands.

Overall JF, Gorham DR (1962). The brief psychiatric rating scale. Psychol Rep 10: 799-812.

Pell MD (2006). Judging emotion and attitudes from prosody following brain damage. Prog Brain Res 156: 303-317.

Potkin SG, Alva G, Fleming K, Anand R, Keator D, Carreon D et al (2002). A PET study of the pathophysiology of negative symptoms in schizophrenia. Positron emission tomography. Am J Psychiatry 159: 227-237.

Provencher SW (2001). Automatic quantitation of localized in vivo 1H spectra with LCModel. NMR Biomed 14: 260-264.

Randolph C (1988). RBANS Manual-Repeatable Battery for the Assessment of Neuropsychological Status. Tex, Psychological Corp (Harcourt): San Antonio.

Shin YW, Kwon JS, Ha TH, Park HJ, Kim DJ, Hong SB et al (2006). Increased water diffusivity in the frontal and temporal cortices of schizophrenic patients. Neuroimage 30: 1285-1291.

Sigmundsson T, Maier M, Toone BK, Williams SC, Simmons A, Greenwood K et al (2003). Frontal lobe $\mathrm{N}$-acetylaspartate correlates with psychopathology in schizophrenia: a proton magnetic resonance spectroscopy study. Schizophr Res 64: 63-71.

Sigmundsson T, Suckling J, Maier M, Williams S, Bullmore E, Greenwood K et al (2001). Structural abnormalities in frontal, temporal, and limbic regions and interconnecting white matter tracts in schizophrenic patients with prominent negative symptoms. Am J Psychiatry 158: 234-243.

Skelly LR, Calhoun V, Meda SA, Kim J, Mathalon DH, Pearlson GD (2008). Diffusion tensor imaging in schizophrenia: relationship to symptoms. Schizophr Res 98: 157-162.
Tamminga CA, Thaker GK, Buchanan R, Kirkpatrick B, Alphs LD, Chase TN et al (1992). Limbic system abnormalities identified in schizophrenia using positron emission tomography with fluorodeoxyglucose and neocortical alterations with deficit syndrome. Arch Gen Psychiatry 49: 522-530.

Thoma RJ, Hanlon FM, Moses SN, Ricker D, Huang M, Edgar C et al (2005). M50 sensory gating predicts negative symptoms in schizophrenia. Schizophr Res 73: 311-318.

Uranova NA, Orlovskaya DD (1996). Ultrastructural pathology of neuronal connectivity in postmortem brains of schizophrenic patients, in Annals of Psychiatry: Basic and Clinical Neurosciences, vol 6. Edited by Cacabelos R. Barcelona, Prous. Science, pp 56-72.

Uranova N, Orlovskaya D, Vikhreva O, Zimina I, Kolomeets N, Vostrikov V et al (2001). Electron microscopy of oligodendroglia in severe mental illness. Brain Res Bull 55: 597-610.

Uranova NA, Vostrikov VM, Orlovskaya DD, Rachmanova VI (2004). Oligodendroglial density in the prefrontal cortex in schizophrenia and mood disorders: a study from the Stanley Neuropathology Consortium. Schizophr Res 67: 269-275.

Uranova NA, Vostrikov VM, Vikhreva OV, Zimina IS, Kolomeets NS, Orlovskaya DD (2007). The role of oligodendrocyte pathology in schizophrenia. Int J Neuropsychopharmacol 10: 537-545.

Vostrikov VM, Uranova NA, Orlovskaya DD (2007). Deficit of perineuronal oligodendrocytes in the prefrontal cortex in schizophrenia and mood disorders. Schizophr Res 94: 273-280.

Wakana S, Jiang H, Nagae-Poetscher LM, van Zijl PC, Mori S (2004). Fiber tract-based atlas of human white matter anatomy. Radiology 230: 77-87.

Wolkin A, Choi SJ, Szilagyi S, Sanfilipo M, Rotrosen JP, Lim KO (2003). Inferior frontal white matter anisotropy and negative symptoms of schizophrenia: a diffusion tensor imaging study. Am J Psychiatry 160: 572-574. 